\title{
Pasting Properties of Composite of Cassava and Wheat Flours
}

\author{
C. Imoisi ${ }^{1 *}$, J.U. Iyasele ${ }^{1}$, E.E. Imhontu ${ }^{2}$, D.O. Ikpahwore ${ }^{1}$ and A.O. Okpebho ${ }^{1}$ \\ ${ }^{1}$ Department of Chemistry, University of Benin, Benin City, Nigeria. P.M.B. 1154, Benin City, Nigeria. \\ ${ }^{2}$ Department of General Studies, Edo State School of Health Technology, Benin City, Edo State, Nigeria \\ *Correspondence Author: imoisi.chinyere@gmail.com, Phone: 07030746386 \\ Received 10 September 2020; accepted 17 October 2020, published online 30 October 2020
}

\begin{abstract}
Classification of cassava and wheat using only amylose content is not sufficient enough to predict starch viscosity for end product recommendation, hence this study aimed at characterizing and categorizing the pasting profile of composite flours from cassava-citrus and wheat-watermelon using Rapid Visco Analyzer (RVA) (used in confectionary products), dried cassava, citrus rind, wheat grain and watermelon rind were processed into flour by grinding. Cassava-citrus and wheat-watermelon flours were blended in the ratio of 100:0, 25:75, 50:50 and 75:25 and labeled as $\mathrm{AB}_{1}, \mathrm{AB}_{2}, \mathrm{AB}_{3}, \mathrm{AB}_{4}$ and $\mathrm{BC}_{1}, \mathrm{BC}_{2}, \mathrm{BC}_{3}, \mathrm{BC}_{4}$ respectively. The data obtained from the pasting properties of composite cassava-citrus and wheat-watermelon flours are as follows: range of peak viscosity (249.6-446.0) and (97.1-116.3) RVU, trough value (158.5-251.5) and (59.570.3) RVU, breakdown viscosity (91.1-184.1) and (37.6-46.0) RVU, final viscosity (222.3-509.3) and (184.4-214.2) RVU, setback viscosity (63.3-247.8) and (124.9-143.8) RVU, peak time (4.27-5.27) and (5.535.67) $\mathrm{min}$ and pasting temperatures (72.45-73.40) and (90.40-91.25) ${ }^{\circ} \mathrm{C}$. The pasting properties of wheat watermelon composite flours increased with increasing substitution of watermelon fibre while those of cassava citrus composite flours decreased with increasing substitution of citrus fibre until $50 \%$ replacement. Composite Cassava-citrus flour $\mathrm{AB}_{3}$ and wheat-watermelon flour $\mathrm{BC}_{1}$ respectively had the highest value for all the pasting properties evaluated. Hence, cassava-citrus flour $\mathrm{AB}_{3}$ with $50 \%$ citrus substitution and wheatwatermelon flour $\mathrm{BC}_{1}$ without any substitution could find applications in confectionery and pastry industries. Thus, the results also indicated that by incorporating citrus and watermelon fibre, it is possible to enhance the pasting properties of our local cassava and wheat flour. This will also serve as a way of converting wastes from citrus (citrus vesicles) and Watermelon rinds (Citrullus larnatus) into useful materials, thereby reducing environmental pollutions caused by these wastes.
\end{abstract}

Keyword: Composite flour, peak viscosity, trough viscosity, breakdown viscosity, final viscosity, setback value.

\section{Introduction}

Consumer have shown a recent tendency towards eating foods that takes less time and effort such as frozen dinners, fast food and snacks. These are food sources that are not served with a necessary amount of dietary fibre. Furthermore, the majority of these foods are prepared from refined wheat flour that is deprived of healthy components like brany and fibery fractions coming from wheat kernel itself [1]. Therefore most consumer donot consume enough level of fiber which their bodies need to work efficiently and feel comfortable with. This is the case for even people who want to make their diets healthy and balanced but still do not get enough fiber on daily basis [1]. Food with low fibre contents are been linked to the emergence of several chronic diseases been demonstrated by some epidemiological researches on colon cancer, and several other diseases [2], leading to the recommendation of dietary fibre in daily diet [3]. For these reasons, improvement of nutritional properties of food has been one of the main interest of researchers. One of the ways to increase nutritional properties can be the incorporation of dietary fibers into food formulations.

Despite many healthy beneficial effects of dietary fibers, their usage in the formulation of food products depends on their functionality as well as their interaction with other ingredients in food formula, which poses some limitation in their usage [4]. Achieving desired functionality in food products is another main concern; therefore; it is important to know the possible interactions of the dietary fiber with main components of food products, which could play a key role on the functionality of the final products. For example, one of the main components widely present in 
formulation of the food product is starch. Starch is added in food product as a thickener, texture improver, stabilizer and in controlling consistency and water retention [4]. Starch achieves these functionalities by affecting pasting, gelatinization and retrogradation properties of many foods [5]. It is known that these properties of starch are markedly affected by some additives [5]. In this respect, dietary fibres are among the most preferred additives that can be used in food industry to improve functionality of starch products [6]. Therefore, the behavior of the starch in the fiber containing medium should be very important since starch is widely used ingredient in the food industry for different purposes.

Composite flours are also blends of wheat and other flours used for the production of leavened breads, unleavened baked products, pastas,

\section{Materials and Methods \\ Sample Collection and Analysis}

Wheat was purchased at Uselu and new Benin local market, Benin City, $7 \mathrm{~kg}$ wheat was weighed with a weighing balance in the laboratory and washed severally before soaking with 7 litres of water overnight, thereafter, the wheat was grinded with a grinder and subsequently sieved with a soft cloth sieve. The sieved wheat was sundried for seven days, thereafter; it was grinded again and subsequently sieved with a smaller sieve to obtain a fine wheat flour powder [8].

Watermelon was purchased at new Benin local market, the pulp was removed to obtain the rind (peel). The Watermelon rinds were thoroughly washed under tap water and then sliced into pieces with stainless steel knives on trays and sundried for 7 days. Grinding in an electric mill before using a grinder to obtain fine rind powder of larger surface area. The fine powder was then stored in a closed container until experiment commenced [9].

The oranges were purchased from Oba Market in Benin City. They were washed with water to remove the contaminants, peeled with a knife and the juice was extracted with a juice extractor. The pectinaceous and cellulosic material called citrus vesicle was blended and washed with hot water. This was done repeatedly for five times to remove the odour, taste, color and acids present in the pulp. It was filtered and sundried for three days at $30-37{ }^{\circ} \mathrm{C}$. The coarse pulp was milled to a very porridges, snacks foods and cakes [7]. Sometimes, only flour is used as replacement-for example, tortillas and wheat-less bread from sorghum, pastas from sorghum or maize [7]. The use of blends or composite flours $(\mathrm{CF})$, of wheat and other flours for bread making has always occurred in times of scarcity of wheat, from whatever cause, climatic or economic. As ingredients are being blended for the composite flour may be cassava, maize, rice, sorghum, the millets, potato, barley, sweet potato and yam. Composite flours are quite different from the ready-mixed flours familiar to millers and bakers. The objective of this study is to expand the utility of citrus and watermelon fibres by value addition through incorporation with cassava and wheat flour respectively to prepare the composite flours and for their characterization and categorization.

fine powder form and preserved in the refrigerator at $4{ }^{\circ} \mathrm{C}$.

The cassava roots were purchased at Oba Market in Benin City. They were washed with water, peeled and re-washed to remove contaminants. The cassava roots were soaked with warm water for three days at $30-37{ }^{\circ} \mathrm{C}$. It was filtered and sundried at $30-37{ }^{\circ} \mathrm{C}$ for seven days. The coarse flour was milled to smooth cassava flour and preserved in the refrigerator at $4{ }^{\circ} \mathrm{C}$.

\section{Preparation of Cassava Bread Incorporated with Citrus Fibre}

Bread was produced using the straight dough process. Baking was carried out under laboratory conditions to optimize baking conditions. Cassava flour and citrus fibre weighing were carried out on a laboratory scale. Dough was mixed to optimum consistency in a mixer with low speed $85 \mathrm{rpm}$ for 1 minute. The replacement of cassava flours by citrus fibers were at various percentages of: $0 \%$, $75 \%, 50 \%$ and $25 \%$ and labeled as $\mathrm{AB}_{1}, \mathrm{AB}_{2}, \mathrm{AB}_{3}$ and $\mathrm{AB}_{4}$ respectively. The cassava flour, citrus fibre and other ingredients for bread were accurately added and the sugar and yeast were mixed in a mixing machine for 20 minutes to produce a cream. The mixed cream was then put into medium size round calibrated pans. All the breads were baked in an oven for 1 hour at 100 ${ }^{\circ} \mathrm{C}$. 


\section{Preparation of Wheat Bread Incorporated with Watermelon Rind Fibre}

Bread was produced using the straight dough process. Baking was carried out under laboratory conditions to optimize baking conditions. Wheat flour and watermelon rind fiber weighing were carried out on a laboratory scale. The dough was mixed to optimum consistency in a mixer with low speed $85 \mathrm{rpm}$ for 1 minute. The replacement of wheat flours by watermelon rind fibers were at various percentages of: $0 \%, 75 \%, 50 \%$ and $25 \%$ and labeled as $\mathrm{BC}_{1}, \mathrm{BC}_{2}, \mathrm{BC}_{3}$ and $\mathrm{BC}_{4}$ respectively. The wheat flour, watermelon rind fiber and other ingredients for bread were accurately added and the sugar and yeast were mixed in a mixing machine for 20 minutes to produce a cream. The mixed cream was then put into medium size round calibrated pans. All the breads were baked in an oven for 1 hour at 100 ${ }^{\circ} \mathrm{C}$.

\section{Pasting Properties Determination}

The pasting properties of the flour samples were determined using the rapid visco analyzer (RVA) according to (Newport scientific, Narrabeen Australia ) as described by Ikegwu et al. [10]. In a typical experiment, $3.50 \mathrm{~g}$ of the flour was mixed with $25.0 \mathrm{ml}$ of distilled water with continuous paddling to form a paste. The samples were then inserted in the rapid visco analyzer. The analysis was carried out at a programmed heating and cooling circle where the samples were held at 50 ${ }^{\circ} \mathrm{C}$ for 1 minute, heated at $95{ }^{\circ} \mathrm{C}$ for 3.8 minutes and held at $50{ }^{\circ} \mathrm{C}$ for 1.4 minutes. The pasting performance of the flour samples was automatically recorded on the graduated sheet of the instrument.

\section{Statistical Analysis}

The data obtained were made in triplicates and analysed using one way analysis of variance (ANOVA) of Statistical Package for Social Sciences (SPSS version 17.0). Significant means were separated using the New Duncan Multiple Range Test (NDMRT) at $95 \%$ confidence interval.

\section{Results and Discussion}

Table 1: Experimental Design

SAMPLE CODE $\quad$ CLASSIFICATION

\begin{tabular}{ll}
\hline $\mathrm{AB}_{1}$ & Control (100g cassava flour) \\
$\mathrm{AB}_{2}$ & $25 \%$ cassava flour $+75 \%$ citrus fibre \\
$\mathrm{AB}_{3}$ & $50 \%$ cassava flour $+50 \%$ citrus fibre \\
$\mathrm{AB}_{4}$ & $75 \%$ cassava flour $+25 \%$ citrus fibre \\
\hline
\end{tabular}

Table 2: Experimental Design

\begin{tabular}{cc}
\hline SAMPLE CODE & CLASSIFICATION \\
\hline $\mathrm{BC}_{1}$ & Control (100g wheat flour) \\
$\mathrm{BC}_{2}$ & $25 \%$ wheat flour $+75 \%$ watermelon fibre \\
$\mathrm{BC}_{3}$ & $50 \%$ wheat flour $+50 \%$ watermelon fibre \\
$\mathrm{BC}_{4}$ & $75 \%$ wheat flour $+25 \%$ watermelon fibre \\
\hline
\end{tabular}


Table 3: pasting properties of Composite Cassava-Citrus flour

\begin{tabular}{llllllll}
\hline Sample & $\begin{array}{l}\text { PV } \\
(\mathrm{RVU})\end{array}$ & $\begin{array}{l}\text { Trough } \\
(\mathrm{RVU})\end{array}$ & BD (RVU) & $\begin{array}{l}\mathrm{FV} \\
(\mathrm{RVU})\end{array}$ & $\begin{array}{l}\text { Setback } \\
(\mathrm{RVU})\end{array}$ & PT(MIN) & PT $\left({ }^{\circ} \mathrm{C}\right)$ \\
\hline $\mathrm{AB}_{1}$ & 249.6 & 158.5 & 91.1 & 222.3 & 63.3 & 4.27 & 73.40 \\
$\mathrm{AB}_{2}$ & 392.8 & 247.3 & 145.5 & 442.5 & 195.3 & 5.13 & 72.50 \\
$\mathrm{AB}_{3}$ & 446.0 & 261.5 & 184.5 & 509.3 & 247.8 & 5.13 & 72.45 \\
$\mathrm{AB}_{4}$ & 376.3 & 260.8 & 115.4 & 479.6 & 218.3 & 5.27 & 72.55 \\
& & & & & & & \\
\hline
\end{tabular}

Table 4: pasting properties of Composite Wheat-Watermelon flour

\begin{tabular}{llllllll}
\hline Sample & PV(RVU) & $\begin{array}{l}\text { Trough } \\
(\mathrm{RVU})\end{array}$ & $\begin{array}{l}\mathrm{BD} \\
(\mathrm{RVU})\end{array}$ & $\mathrm{FV}(\mathrm{RVU})$ & $\begin{array}{l}\text { Setback } \\
(\mathrm{RVU})\end{array}$ & $\begin{array}{l}\mathrm{PT} \\
(\mathrm{MIN})\end{array}$ & $\mathrm{PT}\left({ }^{\circ} \mathrm{C}\right)$ \\
\hline $\mathrm{BC}_{1}$ & 116.3 & 70.3 & 46.0 & 214.2 & 143.8 & 5.67 & 90.55 \\
$\mathrm{BC}_{2}$ & 113.25 & 68.6 & 44.7 & 206.5 & 137.9 & 5.67 & 90.40 \\
$\mathrm{BC}_{3}$ & 104.6 & 64.8 & 39.8 & 190.9 & 126.1 & 5.67 & 91.25 \\
$\mathrm{BC}_{4}$ & 97.1 & 59.5 & 37.6 & 184.4 & 124.9 & 5.53 & 91.20 \\
\hline
\end{tabular}

The pasting properties are important as it is used in predicting the pasting behavior and ability of the flour samples. Pasting properties are the most commonly assessed set of quality characteristics probably because the methods are well established and have been proven to be a reliable predictor of flour quality. The pasting properties are important as it is used in predicting the pasting behavior and ability of the flour samples. Tables 3 and 4 showed the pasting profile of composite cassavacitrus and wheat-watermelon flours respectively. Peak viscosity (PV) indicates the water-holding capacity of the starch or mixture. The rate of breakdown in viscosity to holding strength, hot paste viscosity, depends on the temperature and degree of mixing, the shear stress, applied to the mixture, and the nature of the material itself. The peak viscosity is indicative of the viscous load likely to be encountered during mixing. The higher the peak viscosity the higher the swelling index, while low peak viscosity is indicative of higher solubility as a result of starch degradation or dextrinization, this is also in line with the work of Shittu et al. [11]. The peak viscosity increased with increasing level of citrus fibre substitution. This increase may be attributed to the high starch content of the citrus fibre causing a high gelatinization and swelling index. PV of 
composite cassava citrus flours ranged from 249.6 to $446.0 \mathrm{RVU}$, the highest was $\mathrm{AB}_{3}$ and the lowest was $\mathrm{AB}_{1}$ while the $\mathrm{PV}$ value of composite wheat watermelon flours ranged from 97.1 to 116.3 RVU. The highest value was recorded for $\mathrm{BC}_{1}$, while the lowest value was recorded for $\mathrm{BC}_{4}$. The peak viscosity is indicative of the strength of pastes, which are formed from gelatinization during processing in food applications. It also reflects the extent of granule swelling [12]. It also provides an indication of the viscous load likely to be encountered during mixing. The relatively low peak viscosity in composite flour indicates that the flour may be suited for products requiring low gel strength and elasticity. The PV is measuring the ability of paste to withstand breakdown during cooling. The variation of peak viscosity often associated with the swelling power of starch and the rate of disruption of starch granules [13].

The trough values (TV) or holding strength of composite flour is the minimum viscosity after the peak, making the starch granules of the flour undisrupted when the flour paste is subjected to a holding period of constant temperature, time and shear stress [14]. Hold viscosity measures the viscosity when the swelled starch granules were disrupted upon shearing and heating. Hold viscosity reflect different susceptibility of starch to shear distingration when swelled, especially in starches with lower amylose content. The holding strength of cassava citrus flours ranged from 158.5 to $261.5 \mathrm{RVU}$, the holding strength was highest in $\mathrm{AB}_{3}$ and lowest in $\mathrm{AB}_{1}$ while the trough value of wheat watermelon flours ranged from 59.5 to 70.3 RVU. The holding strength was highest in $\mathrm{BC}_{1}(70.3 \mathrm{RVU})$ and lowest in $\mathrm{BC}_{4}$ (59.5 RVU).

Final viscosity (FV) of flour indicates the ability of the flour to form a viscous paste or gel after cooking and as well as the resistance of the paste to shear stress during stirring. It is also ability of starch to form a viscous paste on cooling. The final viscosity of composite cassava citrus flours ranged from 222.25 to $509.3 \mathrm{RVU}$. Sample $\mathrm{AB}_{3}$ (509.3 RVU) had the highest FV compare to the control (222.25) which indicated the reassociation of starch granules especially amylose during cooling time after gelatinization and the formation of gel network. The increase in final viscosity might be due to aggregation of amylose molecules [15] which is indicative of quick retrogradation [16]. While the final viscosity value of composite wheat watermelon flours ranged from 184.4 to 214.2 RVU. Sample $\mathrm{BC}_{1}(214.2$ RVU) which was the control had the highest FV. Final viscosities are important in determining ability of the flour sample to form a gel during processing.

Setback value is the phase pasting curve after cooling of the starch and this phase involves reassociation, retrogradation or re-ordering of starch molecules. Setback value is the tendency of starch to associate and retrograde on cooling. Peroni et al. [17] indicated that the flour with low setback value may have low values of amylase which have high molecular weight. The lower the retrogradation, the higher the setback value, during cooling of the products made from the flour [18]. High setback value is associated with syneresis. The setback viscosity value of composite wheat watermelon flours ranged from 124.9 to 143.8 RVU. The highest setback viscosity value was recorded for $\mathrm{BC}_{1}(143.8$ $\mathrm{RVU}$ ) while the lowest value was recorded in $\mathrm{BC}_{4}$. The setback viscosity value of composite cassava citrus flours ranged from 63.3 to 247.8 RVU. The highest setback viscosity value was recorded for $\mathrm{AB}_{3}(247.8 \mathrm{RVU})$ while the lowest value was recorded in $\mathrm{AB}_{1}$. There were significant differences among the flour samples for final viscosity and setback. The composite flour could form a much better flour paste that could find application in the confectionaries industry. Set back viscosity indicates gel stability and potential for retrogradation [19]. Chinma et al. [20] also reported that high setback value is an indication of the propensity of the starch molecules to disperse in hot paste and re-associate readily during cooling. The higher setback viscosity with increase in fibre level, indicate less retrogradation level during cooling and the lesser staling of products made from the flour. Setback viscosity was calculated by subtracting peak viscosity from final viscosity. The viscosity changes while cooling were mainly due to amylose molecular reassociation, and low setback viscosity indicated a low rate of starch retrogradation.

Breakdown viscosity is indicative of paste stability [21]. The breakdown value for cassava citrus flours ranged from 37.6 to $46.1 \mathrm{RVU}$ with highest value obtained in the recorded for $\mathrm{AB}_{1}$ (46.0 RVU) and lowest in $\mathrm{AB}_{4}$ (37.6 RVU). The 
breakdown viscosity decreased with increasing level of watermelon fibre substitution. There were significant differences among the four flour samples. The breakdown values for cassava citrus flours ranged from 91.08 to 184.5 RVU with sample $\mathrm{AB}_{3}$ having the highest breakdown viscosity value. This implies that the composite could not breakdown on heating and as such can find application in foods processed by heating at high temperatures. Breakdown viscosity is the measure of the tendency of the swollen starch granules to rupture when held at high temperatures and continuous shearing. Breakdown viscosity can also defined as the difference between peak and hold viscosity. Low breakdown viscosity is often associated with low hydration and swelling power and high resistance [13].

The time at which peak viscosity occurred in minutes is termed peak time [22]. Peak time is the time required for the flour to reach full development. It gives an indication of the optimum mixing time in the bakery. Peak time is also the time to the nearest half-minute from the first addition of the water to the development of the dough's consistency (minimum mobility), immediately before indication of weakening. It's also the time needed for the curve to reach maximum dough consistency and is usually the highest point on the curve when the curve is centered on the 500 B.U. line. Peak time value of watermelon wheat flours ranged from 5.53 to 5.67

\section{Conclusion}

Substitution of cassava with citrus and wheat with watermelon affect the pasting properties of composite flour. The inclusion of citrus flour in cassava flour and watermelon flour in wheat flour up to 50 and $10 \%$ respectively could give confectionary product of high quality that is comparable to with $100 \%$ cassava and wheat flour. The inclusion of citrus flour up to $50 \%$ in cassava flour and $0 \%$ watermelon flour in wheat flour could give a confectionery product of high quality that comparable to $100 \%$ cassava and wheat flour respectively. Composite flour from cassava-citrus and wheat-watermelon flours could be used in the achievement desired in food security where they are abundant. In this it will advance the promotion, utilization and processing of citrus and watermelon rind for improved industrial and domestic use. minutes. While that of cassava citrus flours ranged from 4.27 to 5.27 minutes. Low peak time is indicative of its ability cook fast.

The pasting temperature (PT) is an indication of the minimum temperature required to cook or gelatinize the flour. The ability of starch to imbibe water and swell is primarily dependent on the pasting temperature. Pasting properties indicate the tendency to form paste, the higher the pasting temperature, the faster the tendency for paste to be formed. There were no significant differences in pasting temperatures among the various treatments of composite cassava citrus and wheat watermelon flours which ranged from 72.45 to $73.40{ }^{\circ} \mathrm{C}$ and 90.40 to $91.25{ }^{\circ} \mathrm{C}$ respectively and the value were not significantly different from the control. The attainment of the pasting temperature is essential in ensuring swelling, gelatinization and subsequent gel formation during processing. The pasting temperature is the temperature at which the viscosity starts to rise [23]. A higher pasting temperature indicates high water-binding capacity, higher gelatinization tendency and lower swelling property starch based flour due high to degree of associative forces between the starch granules [24]. A high pasting temperature indicates the resistance potential against swelling in the ingredient, which could be correlated to the amount amylose and amylopectin in the ingredient.

\section{Acknowledgement}

My deep appreciation goes to Prof. J.U. Iyasele of the department of Chemistry, University of Benin, Benin City for his impactful, insightful and invaluable contributions.

\section{References}

1. N. Sozer and A.C. Dalgic (2007), Modelling of rheological characteristics of various Spaghetti types. European food research and technology, 225, 183-190.

2. G.R. Beecher (1999), Phytonutrients Roles in Metabolism: Effects on resistance to degenerative processes. Nutritional Review, 37, 3-6.

3. M.A. Eastwood (1992), the physiological effects of dietary fibre: An update. Annual Review of Nutrition, 12, 19-35. 
4. R. Ellis, M. Cochrane, M. Dale, C. Duffus, A. Lynn and I.M. Morrison (1998), Starch production and Industrial use. Journal of the science of food and agriculture, 77, 289-311.

5. J. Blazek, E.P. Gilbert and L. Copeland (2011), Effects of monoglycerides on pasting properties of wheat starch After repeated heating and cooling; Journal of Cereal Science, 54, 151-159. 6. V.E. Gurmeric, M. Dogan, O.S. Toker, E. Senygit and N.B. Ersoz (2012), Application of different multi-criteria Decision techniques to determine optimum flavor of Pre-biotic budding based on sensory analysis. Food and Bioprocess Technology.

7. D.A.V. Dendy (1993), Review of composite flour technology in the context of Tanzania. Present a paper at the Workshop entitled 'Sorghum and millets marketing and utilization' held at Acrusha, Tanzania during 3-5 May, 1993. 8. AOAC (1990), Official Methods of the Association of Official Analytical Chemists, 15th edn, AOAC, Washington DC, USA. Method; 972, 37.

9. AACC (1995), Approved Methods of the American Association of Cereal Chemists, 9th edn, March, St Paul, Minnesota, USA.

10. O.J. Ikegwu, V.N. Nwobasi, M.O. Odoh and N.U. Oledinma (2009), Evaluation of the Pasting and Some Functional Properties of Starch Isolated from Some Improved Cassava Varieties in Nigeria. African Journal of Biotechnology, 8, 2310-2315.

11. T.A. Shittu, O.O. Lasekan, L.O. Sanni and M.O. Oladosu (2001), the effect of drying methods on the Functional and Sensory Characteristics of Pupuru: A fermented Cassava product. ASSET, 1(2), 9-16.

12. X. Liang and J.M. King (2003), Pasting and Crystalline Property Differences of Commercial and Isolated Rice Starch with Added Amino Acids. Journal of Food Science, 68, 832-838.

13. H. Corke, H. Wu, S. Yue and H. Sun (1997), Developing Specialty Starch from new crops: A Case study Using Grain Amaranth. In Cereals: Novel Uses and processes, 95-100.

14. H.A. Bakare (200), Evaluation of Rheological Properties and Baking Performance of Wheat Composite Flour Produced from Iye peeled breadfruit (Artocarpuscomunis) and Cassava. Ph.D Thesis, University of Ibadan, pp. 170.
15. M.J. Miles, V.J. Morris, P.D. Orford and S.G. Ring (1985), the roles of amylase amylopectin in the gelation of Starch. Carbohydrate Research, 135, 271-281.

16. C.Y. Lii, M.L. Tsai and K.H. Tseng (1996), Effect of Amylose Content on Rheological Property of Rice Starch. Cereal Chemistry, 73, 415-420.

17. F.H.G. Peroni, T.S. Rocha and C.M.L. Franco (2006), Some Structural and Physicochemical Characteristics of Tuber and root Starches. Food Science and Technology International, 12, 505510.

18. O.J. Ikegwu, P.E. Okechikwu and E.O. Ekumankana (2010), Physico-Chemical and Pasting Characteristics of Flour and Starch from Achi (Brachystegia eurycoma) seeds. Journal of Food Technology, 8(2), 58-66.

19. L.L. Niba, M.M. Okanga, F.L. Jackson, D.S. Schlimmme and B.W. Li (2001), Physicochemical Properties and Starch Granular Characteristics of Flour from Various Manihot esculenta (Cassava) Genotypes, Journal of Food Science, 67, 20-24.

20. C.E. Chinma, J.O. Abu and J.A. Ojo (2010), Chemical, Functional and and Pasting Properties of Starches from Brown and Yellow Tiger nuts (Cyperus esculentus). Nigerian Food Journal, 28, 1-13.

21.C.T. Akanbi, S. Nazamid and A.A. Adebowale (2009), Functional and Pasting Properties of a tropical Bread fruit (Artoparcus altilis) starch from Ile-Ife, Osun State, Nigeria. International Food Research Journal, 16, 151-157

22. A.A. Adebowale, L.O. Sanmi and S.O. Awonorin (2005), Effect of Texture Modifiers on the Physicochemical and Sensory Properties of Dried Fufu. Food Science and Technology International, 11, 373-382.

23. J.J.M. Swinkels (1985), Composition and Properties of Commercial Native Starches. Starch-starke, 37, 1-5.

24. Y.A. Adebowale, I.A. Adeyemi and A.A. Oshodi (2005), Variability in the Physicochemical, Nutritional and Antinutritional Attributes of six Mucuna Species. Food Chemistry, 89, 37-48. 